\title{
Entwicklungsaufgaben angehender Primarlehrpersonen in Berufspraktischen Studien
}

\author{
Sabine Leineweber $\mathbb{D} \cdot$ Melanie Billich-Knapp · Julia Košinár
}

Eingegangen: 30. April 2021 / Überarbeitet: 27. Juli 2021 / Angenommen: 16. September 2021 / Online publiziert: 13. Oktober 2021

(C) Der/die Autor(en) 2021

Zusammenfassung Basierend auf dem Konzept der Entwicklungsaufgaben wurden im Kontext der Bildungsgangforschung Entwicklungsaufgaben für Lehrpersonen zunächst theoretisch herausgearbeitet und anschliessend über empirische Studien bestätigt/konkretisiert. Für den Berufseinstieg von Lehrer*innen liegt bereits seit Jahren ein Kanonmodell vor, das erst in jüngster Zeit phasenspezifisch erweitert wurde. Es umfasst die Entwicklungsaufgaben Identitätsstiftende Rollenfindung, Adressatenbezogene Vermittlung, Anerkennende Klassenführung und Mitgestaltende Kooperation. Die Ausbildung von Lehrpersonen wurde im Hinblick auf berufsphasenspezifische Anforderungen lange nicht in den Blick genommen. Mit dem hier vorgestellten Projekt wird ausgehend von qualitativem Interview-Datenmaterial die subjektive Bedeutsamkeit von in der berufspraktischen Ausbildung erfahrenen Anforderungen durch Studierende fokussiert. Es konnten fünf Entwicklungsaufgaben identifiziert werden, von denen sich zwei als anschlussfähig an das Kanonmodell für den Berufseinstieg erweisen (Adressatenbezogene Vermittlung und Anerkennende Klassenführung). Die drei weiteren analysierten Entwicklungsaufgaben weisen Unterschiede zum Kanonmodell auf und verdeutlichen damit die berufsbiographische Phasenspezifität (Sich in Ausbildung befinden, Zusammenarbeit mit verschiedenen Akteur*innen, Ein berufliches Selbstverständnis entwickeln). Mit den Ergebnissen wird dem Desiderat der systematischen, empirisch begründeten Ermittlung von Anforderungen begegnet, die sich subjektiv für Studierende in Praktika konstituieren -

\footnotetext{
Sabine Leineweber $(\varangle) \cdot$ Melanie Billich-Knapp · Julia Košinár Institut Primarstufe, Pädagogische Hochschule FHNW, Bahnhofstrasse 6, 5210 Windisch, Schweiz E-Mail: sabine.leineweber@fhnw.ch

Melanie Billich-Knapp

E-Mail: melanie.billichknapp@fhnw.ch

Julia Košinár

E-Mail: julia.kosinar@fhnw.ch
} 
die sich aber nicht zwangsläufig an die hochschulisch definierten Standards und Entwicklungsziele anschliessen müssen.

Schlüsselwörter Professionalisierung - Berufspraktische Studien · Lehrer*innenbildung · Qualitative Forschung · Entwicklungsaufgaben

\title{
Developmental tasks of teacher trainees in practical vocational studies
}

\begin{abstract}
On the basis of the concept of developmental tasks, developmental tasks for teachers were first theoretically identified in the context of the occupational biographical approach and then identified through empirical studies. A canon model for the career entry of teachers is already available for years and has only recently been expanded in a phase-specific manner. It includes the developmental tasks "rolefinding", "adaptive instruction in the demands of teaching", "respectful classroom management" and "cooperation". The practical training in teacher education has not been looked upon in this respect for a long time. The project presented here focuses on the subjective significance of students' requirements experienced by them in practical training on the basis of qualitative interview data. Five developmental tasks could be identified. Two of them are connected to the canon model for teachers during their career entry ("adaptive instruction in the demands of teaching" and "respectful classroom management"). The three other developmental tasks which could be analyzed show differences which illustrate the occupational biographical phase specificity ("undergoing a training situation", "cooperation with various actors", "development of a professional identity"). This is supposed to counter the desiderata of the systematic, empirically founded identification of requirements which are subjectively experienced by students in internships-and do not necessarily follow the university-defined standards and developmental goals.
\end{abstract}

Keywords Professionalization · Practical training - Teacher education - Qualitative research $\cdot$ Developmental tasks

\section{Einleitung}

In den letzten Jahren hat sich die bildungswissenschaftliche Forschung im deutschsprachigen Raum im Hinblick auf die berufspraktische Ausbildung von Lehrpersonen rasant entwickelt; der ersten Phase der Lehrer*innenbildung wird eine zunehmend hohe Aufmerksamkeit gewidmet. So zeigt sich in wachsendem Maße ein Erkenntnisinteresse an der Bedeutung, die Praxisphasen bzw. die berufspraktischen Studien für angehende Lehrpersonen in der Vorbereitung auf den künftigen Beruf haben (z. B. Arnold et al. 2014; Buchreihe der IGSP'). In Zusammenhang damit stehen auch aktuelle Veränderungen, wie die Einführung von Langzeitpraktika während des Studiums und die Verkürzung des Referendariats in vielen deutschen Bundesländern

1 IGSP: Internationale Gesellschaft für Schulpraktische Studien und Professionalisierung; fünf Bände von 2016-2021. 
(vgl. Gröschner und Klaß 2020; Schubarth und Wachs 2020). In einer einphasigen Lehrer*innenbildung, wie sie in der Schweiz existiert, kommt den berufspraktischen Studien ein noch viel ausdrücklicherer Stellenwert zu als in einer zweiphasigen Ausbildung. Denn hier ist eine direkte Einmündung in den Berufseinstieg nach sechs (Kindergarten, Primarstufe) bzw. nach neun Semestern (Sekundarstufe I) vorgesehen, und die berufspraktischen Studienanteile stellen die einzige Gelegenheit der handlungspraktischen Ausbildung dar.

Insbesondere für die Zeit nach dem Studium liegen Untersuchungen vor, die auf die Wahrnehmung und Bearbeitung lehrberuflicher Anforderungen fokussieren und das Referendariat (Košinár 2021, 2014; Dietrich und Faller 2021; Pille 2013), den Berufseinstieg (v.a. Hericks 2006; Keller-Schneider 20202; Keller-Schneider und Hericks 2014) oder spezifische Anforderungssituationen während der Berufstätigkeit untersuchen (z. B. Wittek 2013; Hericks et al. 2018). Jüngst wurde das bestehende Kanonmodell der Entwicklungsaufgaben für den Berufseinstieg in theoretischer Ableitung für die Ausbildungsphase weiterentwickelt (Keller-Schneider 2021), jedoch liegen bisher keine empirischen Arbeiten zur Erfassung subjektiver Anforderungen Studierender in den Schulpraktika vor. Bestehende Studien zur Ausbildung beziehen sich z. B. auf eine deduktive, an lehrer*innenberuflichen Standards und den Rahmenbedingungen des Referendariats orientierte Ebene (Kiel 2012) sowie auf Anforderungen im Lehramtsstudium (Ostermann 2015; Köffler 2015). Die empirische Leerstelle zur Frage der subjektiven Anforderungswahrnehmung und -deutung in den berufspraktischen Studien zu füllen ist das Anliegen der in diesem Beitrag vorgestellten Interview-Studie im Projekt „Anforderungen Studierender in den Berufspraktischen Studien“, das an der Pädagogischen Hochschule Nordwestschweiz (2014-2017) angesiedelt war. Zum Zeitpunkt der Untersuchung durchliefen die Studierenden während ihres dreijährigen Bachelorstudiums vier je vierwöchige Blockpraktika, während derer sie von erfahrenen, für die Begleitung qualifizierten Praxislehrpersonen begleitet werden (Leineweber 2021). Die Ergebnisse des Projekts mündeten in ein „Modell der Entwicklungsaufgaben von Studierenden in den Berufspraktischen Studien“ Mit diesem Beitrag wird dieses Modell erstmals umfassend vorgestellt (Auszüge: Košinár 2019a, b; Košinár und Laros 2018). Dabei wird zunächst eine professionalisierungstheoretische Rahmung vorgenommen (Abschn. 2), bevor Fragestellung (Abschn. 3) und methodisches Vorgehen der Untersuchung (Abschn. 4) dargelegt werden. In Abschn. 5 wird das phasenspezifische Modell der Entwicklungsaufgaben Studierender in den berufspraktischen Studien unter Bezugnahme auf das Kanonmodell vorgestellt. Abschliessend diskutieren wir in Abschn. 6 die Ergebnisse und legen dar, wie das Modell der Entwicklungsaufgaben angewendet und weiter eingesetzt wurde.

\footnotetext{
${ }^{2}$ Die ursprüngliche Veröffentlichung lag im Jahr 2010; wir beziehen uns auf die aktuelle überarbeitete u. ergänzte Fassung als Referenzpublikation.
} 


\section{Entwicklungsaufgaben als Kernkonzept des berufsbiografischen Professionalisierungsverständnisses}

Die Professionalisierungsforschung weist verschiedene Zugänge zur Bestimmung von Professionalität im Lehrberuf auf: Während der strukturtheoretische Zugang „den Strukturkern professionellen Handelns im Vergleich zu anderen Handlungsvollzügen“ (Helsper 2021, S. 103) fokussiert und „das Handeln der Akteur*innen unter prinzipieller Unsicherheit und Unplanbarkeit" (Košinár 2014, S. 21) betrachtet, geht es mit dem kompetenzorientierten Ansatz um die Ermittlung derjenigen „Kompetenzbereiche und Wissensdomänen“ (Terhart 2011, S. 207), die Lehrkräfte für eine erfolgreiche Bewältigung ihres Berufes benötigen. Dem berufsbiografischen Ansatz wird dabei die Funktion einer ,,verbindende[n] Klammer“ (ebd., S. 209) zugesprochen, mit der Professionalität im berufsbiografischen Verlauf betrachtet wird, womit eine „dynamische Entwicklungsperspektive“ (ebd.) in den Vordergrund rückt, die am Lernprozess von Individuen und ihrer jeweiligen Biografie ansetzt. In diesem Bestimmungsansatz von Professionalität im Lehrberuf ist die hier vorgestellte Studie zu verorten. Den theoretischen Rahmen bildet das Konzept der Entwicklungsaufgaben (Havighurst 1972 [1948]), das als „Kernkonzept“ (Hericks 2006, S. 60) der Bildungsgangforschung betrachtet wird. Es wird damit u. a. danach gefragt, ,wie sich spezifische Handlungsanforderungen eines Feldes oder der Gesellschaft als Ganze - konzeptionell gefasst als Entwicklungsaufgaben - in Biographien von Menschen niederschlagen“ (Keller-Schneider und Hericks 2014, S. 391). Sie werden von Hericks (2006) definiert als ,gesellschaftliche Anforderungen an Menschen in je spezifischen Lebenssituationen, die individuell als Aufgaben eigener Entwicklung gedeutet werden können“ (ebd., S. 60). Übertragen auf den beruflichen Kontext lassen sich Entwicklungsaufgaben damit als Bündel von beruflichen Anforderungen verstehen, deren Wahrnehmung und Bearbeitung als ,unhintergehbar“ betrachtet werden (ebd.). Voraussetzung dafür ist ein handelndes Individuum, das in aktiver Auseinandersetzung mit seiner Umgebung (berufliche) Anforderungen bewusst zu Herausforderungen umdeutet und so die Grundlage für die eigene Weiterentwicklung schafft (vgl. ebd.). Für die Professionalisierungsforschung ist das Entwicklungsaufgabenkonzept insofern relevant, als dass die Subjektperspektive im Vordergrund steht und sowohl die individuellen Voraussetzungen von Personen (z. B. Wissensbestände, Fähigkeiten, habituelle Dispositionen) als auch die kontextuellen Rahmenbedingungen berücksichtigt werden. Es setzt bei den subjektiv als bedeutsam wahrgenommenen Anforderungen an (vgl. Keller-Schneider 2020, S. 150), denn nur diejenigen Anforderungen, die ein Individuum für sich als relevant identifiziert, werden von ihm auch bearbeitet (vgl. ebd., S. 150ff.). Dabei können Anforderungen von aussen an ein Individuum herangetragen werden - im Praktikum z. B. durch die Praxislehrperson als zu bearbeitende Aufgabe. Berufliche Anforderungen können sich aber auch situativ entwickeln oder durch individuelle Entwicklungsanliegen des Subjekts entstehen z. B. aufgrund persönlicher Ansprüche an das eigene berufliche Handeln. Gemäss des von Keller-Schneider entwickelten Anforderungsbearbeitungsmodells (ebd., S. 151) werden - von ihr stress- und ressourcentheoretisch begründet - berufliche Anforderungen positiv gedeutet, sofern ein Individuum über ausreichende Ressourcen verfügt. Subjektiv als bedeutsam wahrgenommene Anforderungen werden so 
zu Herausforderungen umgedeutet und bearbeitbar (vgl. ebd.). Mit der Bearbeitung und Bewältigung von Anforderungen findet ein Prozess der beruflichen Weiterentwicklung statt; er ,eröffnet neue Perspektiven; man geht als jemand anderes aus diesem Prozess hervor“ (Hericks et al. 2019, S. 599).

Für den Berufseinstieg von Lehrpersonen liegt seit einigen Jahren ein empirisch geprüftes Kanonmodell von vier Entwicklungsaufgaben vor ${ }^{3}$ :

1. Identitätsstiftende Rollenfindung beinhaltet Anforderungen, die im Zusammenhang damit stehen, ,eine professionelle Identität als Lehrperson“ (Keller-Schneider und Hericks 2011, S. 22) zu entwickeln, die eigenen Ressourcen angemessen einzusetzen, das Verhältnis von Nähe und Distanz auszutarieren und eigene Grenzen anzuerkennen (vgl. ebd., S. 23).

2. Adressatenbezogene Vermittlung bezieht sich auf diejenigen Anforderungen an das Lehrer*innenhandeln, die die Vermittlung ,,von Fachinhalten und kulturellen Sachverhalten“ umfassen (Hericks 2006, S. 63). Hierzu gehören auch die Gestaltung adaptiver Lernzugänge sowie die individuelle Förderung der Lernprozesse der Schüler*innen (vgl. Keller-Schneider und Hericks 2011, S. 22).

3. Anerkennende Klassenführung umfasst Anforderungen, die sich auf einen anerkennenden Umgang mit dem Gegenüber und die wertschätzende Führung der Klasse als Ganzes sowie auch der einzelnen Schüler*innen beziehen (vgl. ebd.).

4. Mitgestaltende Kooperation beschreibt Anforderungen, die sich auf den institutionellen Kontext und dessen Weiterentwicklung sowie die Kooperation im Kollegium beziehen (vgl. ebd.).

Die berufseinstiegsspezifischen Entwicklungsaufgaben bilden strukturtheoretisch begründete Anforderungen und damit die ,typischen Entwicklungsfelder des beruflichen Lehrerhandelns ab, die über die gesamte Berufsbiografie bestehen bleiben: Person, Sache, Schülerinnen/Schüler, Institution“ (Hericks et al. 2019, S. 600; Hervorhebungen i.O.). Mit den Entwicklungsfeldern werden gemäß weiterführenden Überlegungen der Autor*innen berufsphasenübergreifende Entwicklungsaufgaben deutlich (Rolle, Vermittlung, Anerkennung, Institution; vgl. Keller-Schneider und Hericks 2021, S. 11), die sich in berufsphasenspezifischer Ausprägung, in Entwicklungslinien durch die Berufsbiografie von Lehrpersonen hindurch“ ziehen (Hericks et al. 2019, S. 601). Der empirische Beleg dafür stehe zwar noch aus, aber KellerSchneider (2021) leitet auf theoretischer Ebene jüngst aus den empirisch ermittelten Entwicklungsaufgaben für den Berufseinstieg den Entwicklungslinien folgend „Konkretisierungen“ (ebd., S. 81) für die vorangehende Phase der ,schulprakti-

\footnotetext{
3 Vorgelegt wurden die Entwicklungsaufgaben für den Berufseinstieg von Lehrpersonen von U. Hericks (2006) und M. Keller-Schneider (2010) in je eigenen Studien: Hericks: Rekonstruktion von Einzelfallstudien mit berufseinsteigenden Sekundarstufen-Lehrer*innen; Keller-Schneider: Inhaltsanalytische Auswertung von Supervisionsprotokollen berufseinsteigender Lehrer*innen der Unter-, Primar- und Sekundarstufe; Überführung analysierter Anforderungen in Fragebogenstudie, faktorenanalytische Bündelung v. Anforderungsbereichen zu Entwicklungsaufgaben. Beide Autor*innen benannten die Entwicklungsaufgaben darin zunächst unterschiedlich; sie wiesen v. a. in den Bereichen Anerkennung und Institution Unterschiede auf. Mit der gemeinsamen Publikationen 2011 liegt eine inhaltlich begründete und begriffliche Abstimmung der Entwicklungsaufgaben für den Berufseinstieg von Lehrpersonen vor, auf die wir uns hier beziehen.
} 
schen Ausbildung“ (ebd.) sowie für die nachfolgende als erfahrene Lehrperson, ab, die in einem ,heuristischen Modell“ (ebd.) dargelegt werden. Dabei werden von ihr folgende Entwicklungsaufgaben für die (schulpraktische) Ausbildung abgeleitet: „explorierende Rollenfindung“, ,sachangemessene Vermittlung“, „,kohärente Klassenführung“ und „anschlussfähige Kooperation in und mit der Institution“ (ebd., S. 82). Zentral geht es ihren Beschreibungen nach in der Ausbildungsphase um phasenspezifische anforderungsbezogene Annäherungen, „Erprobungen“ (ebd., S. 82), Auseinandersetzungen und anschlussfähige Nutzung von Erfahrungsräumen (vgl. ebd. $82 \mathrm{ff}$.).

Wir gehen mit unserer Untersuchung davon aus, dass Praktika Entwicklungsaufgaben in berufsphasenspezifischer Ausprägung bereithalten, die strukturiert erfassbar sind. Darum war die Identifikation von für Studierende subjektiv relevanten Anforderungen in den Schulpraktika das zentrale Anliegen der hier vorgestellten Studie.

\section{Fragestellungen der Untersuchung}

Vor dem Hintergrund der dargelegten Rahmung fokussieren wir im vorliegenden Beitrag folgende zwei Fragestellungen:

1. Welchen Anforderungen begegnen angehende Primarlehrpersonen während ihrer berufspraktischen Ausbildung im Studium und inwiefern lassen sich diese (in Entwicklungsaufgaben) bündeln?

2. Inwiefern lassen die identifizierten Anforderungen bzw. Entwicklungsaufgaben eine Ausbildungs-, und somit Berufsphasenspezifität erkennen?

Das Ziel der qualitativen Querschnittsstudie war es Entwicklungsaufgaben während der berufsbiografischen Phase des Studiums zu identifizieren, sie zu beschreiben und mit dem empirisch geprüften Kanonmodell für Lehrpersonen im Berufseinstieg zu vergleichen. Gleichzeitig sollten uns die Ergebnisse für weiterführende Schritte dienen (vgl. Abschn. 6).

\section{Methodisches Vorgehen}

In diesem Abschnitt wird das forschungsmethodische Vorgehen dargelegt. Nach einer Beschreibung von Untersuchungsdesign und -sample wird das Auswertungsverfahren vorgestellt, wobei wir auch unsere Überlegungen und Entscheidungen im Kodierungsprozess verdeutlichen.

\subsection{Untersuchungsdesign und Stichprobe}

Um einen Zugang zum individuellen Erleben und den subjektiven Relevanzsetzungen Studierender zu erhalten, wurden leitfadengestützte, erzählgenerierende Interviews zur Datenerhebung eingesetzt. Die Teilnehmenden wurden in den Interviews u. a. zu positiven sowie herausfordernden Situationen, zu gelungenen/weniger gelungenen Unterrichtsstunden während ihrer Praktika und ihrem Umgang mit (weiteren) 
Herausforderungen in den berufspraktischen Studien befragt. Sie wurden gebeten, einprägsame Entwicklungen und Veränderungen zu schildern, sowie über die $\mathrm{Zu}$ sammenarbeit mit den verschiedenen Beteiligten im Praktikum, genutzte Unterstützung sowie individuelle Besonderheiten oder Bedingungen zu berichten. Der Fokus wurde so auf subjektiv bedeutsame Themen während der Praktika gerichtet, die Hinweise auf Anforderungen sowie den Umgang mit krisenbehafteten Situationen liefern konnten, aber auch auf vorhandene bzw. wahrgenommene Ressourcen. Zur Erfassung möglichst vielfältiger beruflicher Anforderungen, denen angehende Primarlehrpersonen in den berufspraktischen Ausbildungsphasen begegnen, wurden Studierende $(n=25)$ aus allen Praxisphasen des Primarstufenstudiengangs einbezogen, die sich freiwillig zur Teilnahme bereit erklärt hatten. Zum Zeitpunkt der Untersuchung hatten vier der Teilnehmenden gerade das erste Praktikum absolviert, vier das zweite Praktikum, drei hatten die dritte Praxisphase beendet und 14 hatten das vierte Praktikum abgeschlossen und standen somit kurz vor Studienende und Berufseinstieg.

\subsection{Vorgehen bei der Datenauswertung}

Die Auswertung des Interview-Datenmaterials erfolgte nach einer Volltranskription mittels inhaltsanalytischen Vorgehens (Mayring 2010) in mehreren Schritten der induktiven Kategorienbildung. ${ }^{4}$ Das Ziel der Auswertung war die strukturierte Erfassung von Anforderungen möglichst in ihrer gesamten Breite, weshalb kein theoretisch abgeleitetes Kategoriensystem an das Material angelegt wurde. Das Kanonmodell aus dem Berufseinstieg wurde explizit nicht als Auswertungsfolie beigezogen, um eine möglichst grosse Offenheit für die Daten zu gewährleisten (vgl. Böhm 2009, S. 477). Da das Modell zwei von drei Rater*innen zum Zeitpunkt der Auswertung unbekannt war, war es möglich, die Analyse von Anforderungen aus dem Datenmaterial möglichst unvoreingenommen zu realisieren.

Eine zentrale Überlegung bei der Entwicklung des Kodiersystems war es zu definieren, was überhaupt als Anforderung gewertet werden kann. Abgeleitet aus der theoretischen Grundlegung (Abschn. 2) wurden nur solche Textpassagen als Anforderung kodiert, die sich als subjektiv bedeutsam für die Befragten identifizieren liessen. Dabei musste jeweils deutlich werden, dass die Anforderung eine Anstrengungsbereitschaft und/oder subjektiv wahrgenommene Herausforderung beinhaltet. Beschreibungen von Aufgaben im Praktikum (z. B. ,im dritten Praktikum musste man ja die Planungen immer frühzeitig abgeben") wurden hingegen nicht als Anforderung gewertet. Folgende Zitate zeigen Ankerbeispiele, in denen Anforderungen erkennbar sind und wie sie kodiert wurden:

- „Was für mich immer sehr schwierig ist [...], ist das Auftreten vor der Klasse, wenn ich neu vor die Klasse komme.“ (AK_P3_214-215) wurde kodiert als „Die Führung der Klasse als Lerngruppe übernehmen“.

\footnotetext{
${ }^{4}$ Computergestützte Datenanalyse mit MAXQDA.
} 
- „So halt einfach [...] einschätzen, wie viel Zeit sie brauchen eine Aufgabe zu lösen, so, das finde ich extrem schwer.“ (PS_P4_155-156) wurde kodiert als „Arbeitstempo SuS einschätzen“"

Es wurden Kodierregeln erarbeitet, um das Auswertungsvorgehen mit mehreren Rater*innen regelgeleitet durchzuführen, Trennschärfe in den Kodierungen herzustellen und intersubjektive Nachvollziehbarkeit zu gewährleisten (vgl. Steinke 2009, S. 324 ff.). So wurde z. B. Eindeutigkeit bei der Kodierung priorisiert, d. h. Doppelkodierungen wurden vermieden, es wurden je Anforderungskategorie Ankerbeispiele beschrieben und festgehalten, inwiefern Kontextinformationen in die Kodierung eingehen sollten. Da eine Quantifizierung der Ergebnisse nicht angestrebt wurde, wurde der Auswertungsprozess kommunikativen Validierungsverfahren unterzogen und hierfür regelmässig im (auch erweiterten) Auswertungsteam validiert. Durch diese Prüfung der Einordnung von am Projekt nicht beteiligten Kolleg*innen wurde eine kritische (Fremd-)Perspektive auf Daten und Auswertung ermöglicht. In mehreren Arbeitsschritten entstand ein umfangreiches Kategoriensystem auf verschiedenen Abstraktionsebenen, das wiederholt auf Gemeinsamkeiten und Unterschiede geprüft und ausdifferenziert wurde (vgl. Kuckartz 2010, S. 86). Die kodierten Anforderungen liessen sich zu 75 Unterkategorien bündeln, die zu 23 Anforderungsbereichen gefasst wurden. Diese konnten 5 Hauptkategorien zugeordnet werden, die als Entwicklungsaufgaben in den berufspraktischen Studien beschrieben werden. Sie werden im nächsten Abschnitt vorgestellt.

\section{Entwicklungsaufgaben und Anforderungsbereiche in den berufspraktischen Studien}

Im Folgenden werden die fünf identifizierten Entwicklungsaufgaben in den berufspraktischen Studien mit den dazugehörigen Anforderungsbereichen dargelegt. Um sie direkt in Verbindung zum Kanonmodell der Entwicklungsaufgaben im Berufseinstieg zu setzen und eine Vergleichbarkeit so auch im Lesefluss zu ermöglichen, werden sie jeweils kurz vergleichend dargestellt.

Wie die Gegenüberstellung verdeutlicht, zeigen sich professionsspezifische $\mathrm{Ge}$ meinsamkeiten sowie phasenspezifische Differenzen:

\begin{tabular}{ll}
\hline Entwicklungsaufgaben in den berufspraktischen Studien & Entwicklungssaufgaben im Berufseinstieg \\
\hline 1. Entwicklung eines beruflichen Selbstverständnisses & 1. Identitätsstiftende Rollenfindung \\
2. Adressatenbezogene Vermittlung & 2. Adressatenbezogene Vermittlung \\
3. Anerkennende Klassenführung & 3. Anerkennende Klassenführung \\
4. Zusammenarbeit mit verschiedenen Akteur*innen & 4. Mitgestaltende Kooperation \\
5. Sich in Ausbildung befinden & - \\
\hline
\end{tabular}

Gemeinsamkeiten zeigen sich vor allem in den vermittlungs- und klassenführungsbezogenen Anforderungen. Jedoch bildet sich auch die Spezifik der Ausbildungssituation ab. So zeigt sich z.B., dass Anforderungen an die Rollenfindung sowie die Kooperation in den berufspraktischen Studien nicht in gleicher Form 
auftreten, wie sie für den Berufseinstieg beschrieben werden. Zudem haben wir mit der Kategorie Sich in Ausbildung befinden eine fünfte, ausbildungsphasenspezifische Entwicklungsaufgabe identifiziert.

\subsection{Entwicklung eines beruflichen Selbstverständnisses}

Für die Entwicklung eines beruflichen Selbstverständnisses wurden folgende Anforderungsbereiche erkannt:

Entwicklung eines beruflichen Selbstverständnisses

Die eigene Professionalität weiterentwickeln umfasst Anforderungen an die Nutzung von Lerngelegenheiten und deren Reflexion. Im Zentrum dieses Anforderungsbereiches stehen darüber hinaus die Erweiterung des professionellen Wissens sowie das Ausbalancieren der Diskrepanz, bereits als Lehrperson zu agieren und sich gleichzeitig als Lernende zu begreifen (und entsprechend sichtbar zu werden).

Nach berufsethischen Überzeugungen handeln beinhaltet Anforderungen an das Austarieren des Handelns als Berufsperson in Abgleich zu den eigenen Überzeugungen über den Lehrberuf und die Schulrealität. Hierzu zählen auch Ansprüche an die Gleichbehandlung aller Schüler*innen und einen gleichermassen professionellen Umgang mit allen beteiligten Personen.

Mit Ungewissheit umgehen beschreibt Anforderungen daran, wahrzunehmen und zu akzeptieren, dass Unvorhergesehenes geschieht. Dazu zählt der Anspruch daran, ausreichend flexibel und adäquat auf unerwartete Situationen reagieren zu können (verweist auf Strukturelement der Ungewissheit und Unplanbarkeit lehrberuflichen Handelns).

Die Berufsrolle als Lehrperson wahrnehmen umfasst die Erkundung der Lehrer*innenrolle sowie das Finden eines persönlichen Stils als Lehrperson. Dazu gehört es, das vielfältige Aufgabenspektrum einer Lehrperson wahrzunehmen, ein angemessenes Rollenverhalten auszubilden und die Grenzen der eigenen Zuständigkeit auszuloten.

Im Zentrum der Bereiche stehen Anforderungen an die grundlegende Entfaltung eines ersten Selbstverständnisses Studierender von sich als Lehrperson im komplexen künftigen Berufsfeld. Es zeigt sich darin, dass sich Studierende während der berufspraktischen Ausbildungsphase ,in ihrer Berufserkundung“ (Košinár 2019b, S. 87) befinden und - in geschütztem Rahmen - ,die Vielseitigkeit lehrberuflicher Anforderungen“ (ebd.) erfahren. Der Unterschied zur identitätsstiftenden Rollenfindung im Berufseinstieg liegt hier v. a. im Prozess der Entwicklung eines professionellen Ichs und in einer Auseinandersetzung mit dem eigenen Verständnis von sich als Lehrperson im (künftigen) Tätigkeitsfeld Schule.

\subsection{Adressatengerechte Vermittlung}

Die Entwicklungsaufgabe Adressatengerechte Vermittlung umfasst in den berufspraktischen Studien folgende Anforderungsbereiche: 
Adressatengerechte Vermittlung

Den Lern- und Entwicklungsstand der Schüler*innen einschätzen beinhaltet Anforderungen Studierender daran, die bei Schüler*innen vorhandenen Kompetenzen einschätzen (,Was sie schon alles können, wo

kann ich ansetzen?") sowie die Lern- und Arbeitsgeschwindigkeit der Schüler*innen abwägen zu können (,Wieviel Zeit brauchen sie für einen Arbeitsauftrag?“).

Unterricht planen erfordert von Studierenden, das unterrichtliche Geschehen in seiner gesamten Komplexität soweit zu erfassen, dass die Lerneinheit entlang formulierter Lernziele durchdacht, konkretisiert und ausgestaltet ist, und sie in der Lage sind, dies auch schriftlich abzubilden. Hierzu zählen auch Anforderungen an die Materialbeschaffung, -gestaltung und -bereitstellung.

Unterricht methodisch und didaktisch strukturieren beinhaltet Anforderungen an eine dem Unterrichtsgegenstand angemessene, methodisch abwechslungsreiche Gestaltung und Rhythmisierung der Lektionen.

Differenzierung realisieren bezeichnet Anforderungen daran, niveaudifferenzierten Unterricht zu gestalten und Materialien dementsprechend aufzubereiten.

Klarheit und Verständlichkeit herstellen verweist auf Anforderungen an die adressatengerechte Formulierung von Arbeitsaufträgen und die Verwendung einer altersadäquaten Unterrichtssprache.

Sowohl im Praktikum als auch im Berufseinstieg geht es darum „eine Passung der fachlichen Anforderungen an den Lernprozess der Lernenden zu entwickeln“ (Keller-Schneider und Hericks 2011, S. 23). Die Phasenspezifität zeigt sich darin, dass die Anforderungen an Studierende durch die zeitliche Begrenzung der Praxisphasen moderiert werden. Die kurze Praktikumsdauer erschwert es Studierenden, die Schüler*innen ausreichend kennenzulernen, um Anforderungen der Vermittlung umfassend selbständig bearbeiten zu können. Hier ist oftmals die Unterstützung der Praxislehrperson vonnöten. In Bezug auf die Planung von Unterricht(ssequenzen) müssen Studierende dabei oft eine vorhandene Diskrepanz überwinden, denn in der Praxis treffen sie auf erfahrene Praxislehrpersonen, deren Unterrichtsplanungen i. d. R. in einer anderen Form erfolgen, als es die Hochschule von den Studierenden im Lernprozess verlangt.

\subsection{Anerkennende Klassenführung}

Die Entwicklungsaufgabe Anerkennende Klassenführung beinhaltet - wie im Kanonmodell - Anforderungen an die Führung der Klasse als Ganzes sowie die Anerkennung der Schüler*innen in ihrer Individualität. Dazu zählen folgende Anforderungsbereiche:

\section{Anerkennende Klassenführung}

Die Führung der Klasse als Lerngruppe übernehmen umfasst Anforderungen an Studierende, die Führung der gesamten Klasse angemessen und (innerhalb des vorgegebenen Rahmens) auszugestalten.

Mit herausforderndem Verhalten von Schüler*innen umgehen beinhaltet Anforderungen daran, angemessen auf Störungen/herausforderndes Verhalten zu reagieren, Motive und Auslöser dafür zu erkennen und mit den in der Klasse etablierten Regelungen umzugehen sowie auch eigene Umgangsweisen zu entwickeln.

Allgegenwärtigkeit zeigen stellt Anforderungen an Studierende, sowohl die einzelnen Schüler*innen als auch die Lerngruppe als Ganzes im Blick zu behalten.

Eine Beziehung zu den Schüler*innen entwickeln beinhaltet Anforderungen an eine vertrauensvolle Beziehungsgestaltung mit den Schüler*innen und daran, sie in ihrer Individualität wahrzunehmen und wertzuschätzen. 
Die Phasenspezifität dieser Entwicklungsaufgabe ist zum einen darin zu sehen, dass Studierende in eine Klasse kommen, in der ein Regelsystem besteht, das nicht sie selbst etabliert haben, das sie aber berücksichtigen und anwenden müssen. Dabei fehlt ihnen noch die Erfahrung, um Verhaltensweisen der Schüler*innen angemessen einschätzen und damit umgehen zu können. Zum anderen ist durch die zeitlich begrenzte Praktikumsdauer das Aufbauen einer tragfähigen Beziehung für Studierende besonders anfordernd. In den Daten deutet sich diesbezüglich aber eine Prozesshaftigkeit an: So zeigt sich, dass es Studierenden in späteren Praxisphasen zunehmend leichter fällt, einen Zugang zu den Schüler*innen zu finden, so dass sie durch die Anforderung offensichtlich weniger stark beansprucht werden.

\subsection{Zusammenarbeit mit verschiedenen Akteur*innen}

Mit der Entwicklungsaufgabe Zusammenarbeit mit verschiedenen Akteur*innen kommt der institutionelle Kontext zum Tragen. Im Vergleich mit Berufseinsteigenden liegt der Fokus Studierender in diesem Bereich auf einer gelingenden Zusammenarbeit mit den für sie relevanten Personengruppen und in erster Kontaktaufnahme mit einem erweiterten Kreis (Eltern, Kollegium). Folgende Anforderungsbereiche konnten identifiziert werden:

Zusammenarbeit mit verschiedenen Akteur*innen

Mit der Praxislehrperson zusammenarbeiten umfasst Anforderungen an die Realisierung einer positiven Gestaltung der Zusammenarbeit. Dazu zählt es, die jeweiligen Bedingungen und Erwartungen zu klären, eine angemessene Form der Zusammenarbeit zu etablieren und in einen vertrauensvollen Austausch zu gelangen.

Mit der/dem Tandempartner*in zusammenarbeiten beschreibt Anforderungen an die produktive Gestaltung des Praktikums mit der/dem Tandempartner*in. Dazu zählt es, gegenseitig die jeweiligen Bedingungen und Erwartungen zu klären und Absprachen einzuhalten. Es zeigt sich in diesem Bereich für Studierende auch als Anforderung, im bzw. trotz Tandem je eigene Verantwortung zu übernehmen und angemessen selbstständig/-verantwortlich zu agieren.

Mit weiteren pädagogischen Fachpersonen zusammenarbeiten beinhaltet Anforderungen daran, ersten Kontakt zu weiteren Fachpersonen (z. B. Heilpädagog*in) herzustellen, deren Perspektive wahrzunehmen, sie einbeziehen zu können sowie die unterschiedlichen Tätigkeitsschwerpunkte kennenzulernen und ein Verständnis des erweiterten institutionellen Umfelds zu entwickeln (z. B. hinsichtlich Entscheidungsfindung, Zuständigkeiten, institutionellen Zugehörigkeiten).

Mit Eltern in Kontakt treten beschreibt Anforderungen daran, mit Eltern in Kommunikation über Schule zu gelangen und eine angemessene Form des Austauschs über ihre Kinder zu realisieren (ggf. etablieren). Dies erweist sich für Studierende als herausfordernd, weil es auch darum geht, von Eltern als angehende Lehrperson anerkannt zu werden. Auch zählen Anforderungen hinzu, mit Rückmeldungen u./o. Beschwerden von Eltern umzugehen.

Mit dem Kollegium in Kontakt treten bezeichnet Anforderungen daran, sich dem Kollegium einer Schule anzunähern und am Austausch zu partizipieren. Dabei geht es auch um eine erste systematische Einordnung einer Kollegiumskultur mit dazugehörigen Abläufen und Ritualen.

Die Anforderungen dieser Entwicklungsaufgabe unterscheiden sich deutlich von denen der mitgestaltenden Kooperation im Kanonmodell. Für Studierende geht es darum, mit den für sie zentralen Akteur*innen basale und positive (zeitlich begrenzte) Formen der Zusammenarbeit zu entwickeln und sich gleichzeitig als angehende Lehrpersonen zu zeigen. Erst zum Studienende lässt sich in einigen Interviews die 
Tendenz zur Positionierung als Kolleg*in erkennen. Es konnten im Datenmaterial keine auf die Weiterentwicklung der konkreten Institution Schule bezogene Anforderungen ausgemacht werden. Dies lässt sich über die kurzen Praktikumsaufenthalte an einer Schule erklären, während derer die anderen Entwicklungsbereiche im Vordergrund stehen. ${ }^{5}$

\title{
5.5 Sich in Ausbildung befinden
}

Mit Sich in Ausbildung befinden wurde eine zusätzliche berufsphasenspezifische Entwicklungsaufgabe identifiziert, die v. a. im Rahmen der berufspraktischen Studien hohe Relevanz erfährt. Mit ihr werden Anforderungen zusammengefasst, die durch den strukturellen Kontext der Ausbildungssituation bedingt sind:

\begin{abstract}
Sich in Ausbildung befinden
Sich am Praktikumsplatz orientieren beschreibt Anforderungen an das jeweilige Einfinden in die Organisation und ihre spezifische Kultur, in Strukturen und Gegebenheiten im Schulhaus sowie im Klassenzimmer.

Mit der asymmetrischen Beziehung zur Praxislehrperson umgehen umfasst Anforderungen an den Umgang damit, sich zeitgleich in einem Erfahrungsraum wie in einer Bewährungssituation zu befinden, im Lernprozess begleitet/beraten und gleichzeitig bewertet zu werden und mit Feedback angemessen umzugehen.

In den Handlungsmöglichkeiten eingeschränkt sein beschreibt Anforderungen, die auf verschiedene Zusammenhänge verweisen: (a) Unterrichten in einer bestehenden Unterrichtskultur mit einem implementierten Regelsystem, das zu befolgen ist. (b) Praktikum im Tandem durchführen: Umgang mit Einschränkungen durch wahrgenommene Konkurrenzsituationen (z. B. weil beide Tandempartner*innen Lernsituationen für sich nutzen möchten u./o. sich in Konkurrenz um die „Gunst der Praxislehrperson“ sehen).

Sich in eine Ausbildungsstruktur einfügen verweist ebenfalls auf eine strukturelle Bedingtheit der Ausbildung: Hierzu zählen Anforderungen daran, zeitgleich Aufgaben aus verschiedenen Bereichen (Schule, Hochschule, tw. Erwerbsleben, familiäre Verpflichtungen) erfüllen und ausbalancieren zu müssen.

Den Berufseinstieg meistern umfasst Anforderungen, die sich auf die Auseinandersetzung mit Grenzen des eigenen Könnens, der Antizipation des Berufseinstiegs mit vollumfänglicher Verantwortungsübernahme und den Umgang mit Überforderungsgefühlen richten.
\end{abstract}

Für diese fünfte und berufsphasenspezifische Entwicklungsaufgabe gilt, dass nicht alle Anforderungsbereiche in jeder Praxisphase gleichermassen relevant werden und ihre Bearbeitung in bestimmten zeitlichen Abfolgen auftritt. So konnten Anforderungen des Bereichs Sich am Praktikumsplatz orientieren zwar im Kontext jeder Praxisphase belegt werden, sie beziehen sich aber vor allem auf die Anfangsphase eines Praktikums und verlieren in dessen Verlauf an Relevanz. Obwohl angenommen werden könnte, dass der Anforderungsbereich Mit der asymmetrischen Beziehung zur Praxislehrperson umgehen mit dem Studienverlauf eine zunehmend geringere Herausforderung darstellt, da Studierende die Beziehungsgestaltung zu

\footnotetext{
5 Dieser Befund schliesst an Hericks (2006) an, der in seiner Untersuchung - entgegen seiner Vorannahme - eine Sequenzialität beruflicher Entwicklungsaufgaben feststellen konnte: Es sei ,eine bestimmte Reihenfolge der Wahrnehmung und Bearbeitung von Entwicklungsaufgaben zutage“ (ebd., S. 423) getreten, wobei Anforderungen an die mitgestaltende Kooperation (in seiner Studie noch als "Institution“ bezeichnet), als letzte der vier Entwicklungsaufgaben für die Berufseinsteigenden relevant wurden.
} 
ihren Praxislehrpersonen mit wachsender Erfahrung gezielter auf ihre individuellen Entwicklungsziele hin angehen, bestätigt sich dies nicht.

\section{Diskussion und Verwendung des Modells}

Es konnte mit den vorliegenden empirisch herausgearbeiteten Entwicklungsaufgaben gezeigt werden, dass die von Hericks et al. (2019) strukturtheoretisch begründeten Entwicklungsfelder auch in der berufspraktischen Ausbildung von Relevanz sind. Es wurde aber deutlich, dass die Ausbildungsphase spezifische Anforderungen und Ausdifferenzierungen mit sich bringt: So befinden sich Studierende noch im Modus des Herantastens an die Aufgabenvielfalt und sind dabei durch die kurze Dauer der Praktika in den Handlungsmöglichkeiten eingeschränkt. Insbesondere mit der fünften Entwicklungsaufgabe zeigt sich, dass Studierende mit den strukturellen Gegebenheiten, die die Praxisphasen mit sich bringen, ausbildungsphasenspezifische Anforderungen an sich gestellt sehen, mit denen sie einen Umgang finden müssen. Dabei wird deutlich, dass in der berufspraktischen Ausbildung eine symmetrische Kooperation ,, auf Augenhöhe“ (Fraefel 2018, S. 20f.) mit der Praxislehrperson nicht realisierbar ist, auch wenn sie in mancher Konzeption programmatisch ist (vgl. ebd.). Dies könnte sich z. B. mit der Einführung von Langzeitpraktika verändern, die u.a. stärker auf ko-konstruktive Zusammenarbeitsformen zielen. Erste eigene Erkenntnisse zum 2017 eingeführten einjährigen Partnerschulpraktikum an der PH FHNW zeigen, dass Partizipation an den Lernprozessen der Schüler*innen sowie ein Aufbau erster Routinen und Selbstständigkeit durchaus erkennbar ist, was eine erweiterte Teilhabe an der Verantwortung für den Unterricht ermöglicht (Košinár in Vorbereitung).

Mit den hier vorgestellten Ergebnissen kann auch den jüngsten theoretischen Ableitungen des Kanonmodells (Keller-Schneider 2021) grundsätzlich zugestimmt werden (vgl. Abschn. 2). Kritisch ist dabei anzumerken, dass die ,hergeleiteten Konkretisierungen“ (ebd., S. 81; Hervorhebungen i. O.) für die Ausbildungsphase aus unserer Sicht nicht die volle Komplexität der Anforderungen in den berufspraktischen Studien abbilden. Mit der fünften Entwicklungsaufgabe (Sich in Ausbildung befinden) zeigen unsere Daten, dass die strukturellen Bedingungen der Ausbildungssituation gerade in der Berufspraxis spezifische Anforderungen mit sich bringen, die in den vier Entwicklungsaufgaben im Berufseinstieg keinen Niederschlag finden, sich aber als für Studierende (und auch Referendar*innen) von hoher Relevanz erweisen (vgl. Košinár 2014; Kahlau 2021). Studierende können die Berufsrealität und -komplexität in den Praxisphasen noch nicht voll erfahren. Im Vergleich zum Berufseinstieg unterliegen sie einem Bewertungskontext, in dem fremde Erwartungen erfüllt, fremde Strukturen und Rituale ,übernommen“ und beibehalten werden müssen. Dabei werden gleichzeitig Anforderungen und Ansprüche aus zwei Ausbildungsinstitutionen (Praktikumsschule, Hochschule) relevant, die nicht immer widerspruchsfrei, aber von Studierenden auszubalancieren und zu bearbeiten sind.

Das hier vorgestellte Modell der Entwicklungsaufgaben in den berufspraktischen Studien ging in die konzeptionellen Überlegungen des Studienbereichs Berufspraktische Studien am Institut Primarstufe der PH FHNW ein (Košinár 2018). So wur- 
den entlang des Modells praxisphasenspezifische Entwicklungsziele definiert und in den Praktikumsleitfäden konkrete Vorschläge zur Bearbeitung der den Entwicklungsaufgaben inhärenten Anforderungen und Unterstützungsmöglichkeiten durch die Ausbilder*innen ausgeführt. Auch wurde auf Grundlage der identifizierten Anforderungen ein Beobachtungs- und Feedbackbogen entwickelt, der eine kriterienbezogene Rückmeldung durch die Praxislehrperson und eine Fokussierung zu bearbeitender Anforderungsbereiche in den Praxisphasen über das Studium hinweg ermöglicht. Das von uns vorgelegte Entwicklungsaufgabenmodell diente ferner als Heuristik von Professionalitätskategorien, die Košinár und Laros (2018) an die Relationale Typologie von Studierendentypen anlegten. Über Einzelfälle konnten sie nachzeichnen, welche Studierendentypen welche Entwicklungsaufgaben bearbeiten bzw. vermeiden. Es konnte eine ,typenbezogene Relevanzsetzung bzgl. der wahrgenommenen und bearbeiteten Anforderungen“ (ebd., S. 169) ausgemacht werden, die belegt, dass insbesondere jene Studierende, die das Praktikum als „Bewertungsraum" rahmen, der Entwicklungsaufgabe sich in Ausbildung befinden die grösste Aufmerksamkeit schenken. Wird das Praktikum als Entwicklungsraum gerahmt, werden hingehen Anforderungen der Entwicklungsaufgaben Ein berufliches Selbstverständnis entwickeln, der Klassenführung und Vermittlung systematisch bearbeitet. Weiter dienten die identifizierten Anforderungen zur Entwicklung eines Fragebogens für die Evaluation der Praxisphasen in unserem Institut. Dieser erfasst die Deutung und Bearbeitung von Anforderungen in den fünf Entwicklungsaufgaben sowie die damit einhergehende Beanspruchung Studierender. In verschiedenen Kohorten eingesetzt lassen sich damit u. a. Veränderungen in der Selbsteinschätzung Studierender bzgl. der wahrgenommen Relevanz und Bearbeitung von Anforderungen in den verschiedenen Entwicklungsaufgaben über den Studienverlauf hinweg zeigen (BillichKnapp 2019; Billich-Knapp et al. 2018). Zusammengefasst konnte ein empirisch begründetes Entwicklungsaufgabenmodell vorgelegt werden, das seitdem verschiedene Funktionen erfüllt, die gesamthaft zu konzeptuellen Weiterentwicklungen der Berufspraktischen Studien beitragen konnten, und das Eingang in Fortbildungen von Dozierenden und Praxislehrpersonen gefunden hat.

Funding Open access funding provided by FHNW University of Applied Sciences and Arts Northwestern Switzerland

Open Access Dieser Artikel wird unter der Creative Commons Namensnennung 4.0 International Lizenz veröffentlicht, welche die Nutzung, Vervielfältigung, Bearbeitung, Verbreitung und Wiedergabe in jeglichem Medium und Format erlaubt, sofern Sie den/die ursprünglichen Autor(en) und die Quelle ordnungsgemäß nennen, einen Link zur Creative Commons Lizenz beifügen und angeben, ob Änderungen vorgenommen wurden.

Die in diesem Artikel enthaltenen Bilder und sonstiges Drittmaterial unterliegen ebenfalls der genannten Creative Commons Lizenz, sofern sich aus der Abbildungslegende nichts anderes ergibt. Sofern das betreffende Material nicht unter der genannten Creative Commons Lizenz steht und die betreffende Handlung nicht nach gesetzlichen Vorschriften erlaubt ist, ist für die oben aufgeführten Weiterverwendungen des Materials die Einwilligung des jeweiligen Rechteinhabers einzuholen.

Weitere Details zur Lizenz entnehmen Sie bitte der Lizenzinformation auf http://creativecommons.org/ licenses/by/4.0/deed.de. 


\section{Literatur}

Arnold, K.-H., Gröschner, A. \& Hascher, T. (2014). Schulpraktika in der Lehrerbildung. Theoretische Grundlagen, Konzeptionen, Prozesse und Effekte. Münster: Waxmann.

Billich-Knapp, M. (2019). Die Partnerschulphase auf dem Prüfstand: Rekonstruktion und Evaluation studentischer Entwicklung im Längsschnitt: Lernen im Partnerschuljahr. Vortrag, 3. Tagung der IGSP, Graz.

Billich-Knapp, M., Košinár, J., \& Leineweber, S. (2018). Entwicklungsaufgaben Studierender in den Berufspraktischen Studien. Einschätzung subjektiv bedeutsamer Anforderungen und deren Bearbeitung im Längsschnitt. Posterpräsentation, 26. Kongress der DGfE, Essen.

Böhm, A. (2009). Theoretisches Kodieren: Textanalyse in der Grounded Theory. In U. Flick, E. von Kardorff \& I. Steinke (Hrsg.), Qualitative Forschung. Ein Handbuch (S. 475-485). Reinbek: Rowohlt.

Dietrich, F., \& Faller, C. (2021). Halbierte Professionalisierung. Eine krisentheoretische Rekonstruktion der Ausbildungslogik des Referendariats. In J. Peitz \& M. Harring (Hrsg.), Das Referendariat (S. 83-92). Münster: Waxmann.

Fraefel, U. (2018). Hybride Räume an der Schnittstelle von Hochschule und Schulfeld. In L. Pilypaitytė \& H.-S. Siller (Hrsg.), Schulpraktische Lehrerprofessionalisierung als Ort der Zusammenarbeit (S. 13-43). Wiesbaden: Springer.

Gröschner, A. \& Klaß, S. (2020). Praxissemester und Langzeitpraktikum. In Cramer, C., König, J., Rothland, M. \& Blömeke, S. (Hrsg.), Handbuch Lehrerinnen- und Lehrerbildung (S. 629-635). Bad Heilbrunn: Klinkhardt (utb).

Havighurst, R. J. (1972). Developmental tasks and education (3. Aufl.). New York, London: Longman.

Helsper, W. (2021). Professionalität und Professionalisierung pädagogischen Handelns: Eine Einführung. Opladen u.a.: Budrich.

Hericks, U. (2006). Professionalisierung als Entwicklungsaufgabe. Rekonstruktionen zur Berufseingangsphase von Lehrerinnen und Lehrern. Wiesbaden: VS.

Hericks, U., Rauschenberg, A., Sotzek, J., Wittek, D., \& Keller-Schneider, M. (2018). Lehrerinnen und Lehrer im Berufseinstieg. Eine mehrdimensionale Typenbildung zu Spannungsverhältnissen zwischen Habitus und Normen. In R. Bohnsack, N.F. Hoffmann \& I. Nentwig-Gesemann (Hrsg.), Typenbildung und Dokumentarische Methode. Forschungspraxis und methodologische Grundlagen (S. 51-67). Opladen: Budrich.

Hericks, U., Keller-Schneider, M., \& Bonnet, A. (2019). Lehrerprofessionalität in berufsbiographischer Perspektive. In M. Harring, C. Rohlfs \& M. Gläser-Zikuda (Hrsg.), Handbuch Schulpädagogik (S. 111-121). Münster: Waxmann.

Kahlau, J. (2021). (De-)Professionalisierung durch Schulpraxis-Erfahrungen. Unveröff. Diss. Bremen: Universität Bremen.

Keller-Schneider, M. (2010). Entwicklungsaufgaben im Berufseinstieg von Lehrpersonen. Münster u.a.: Waxmann.

Keller-Schneider, M. (2020). Entwicklungsaufgaben im Berufseinstieg von Lehrpersonen (2. Aufl.). Münster: Waxmann.

Keller-Schneider, M. (2021). Entwicklungsaufgaben aus entwicklungspsychologischer sowie aus stressund ressourcentheoretischer Perspektive als Zugang zur Professionalisierung von Lehrpersonen. In T. Leonhard, P. Herzmann \& J. Košinár (Hrsg.), „, Grau, theurer Freund ist alle Theorie “? Theorien und Erkenntnisweg Schul- und Berufspraktischer Studien (S. 73-89). Münster: Waxmann.

Keller-Schneider, M., \& Hericks, U. (2011). Beanspruchung, Professionalisierung und Entwicklungsaufgaben im Berufseinstieg von Lehrerinnen und Lehrern. Journal für Lehrerinnen- und Lehrerbildung, 2(11), 20-31.

Keller-Schneider, M., \& Hericks, U. (2014). Forschungen zum Berufseinstieg. Übergang von der Ausbildung in den Beruf. In E. Terhart, H. Bennewitz \& M. Rothland (Hrsg.), Handbuch der Forschung zum Lehrerberuf (S. 386-407). Münster: Waxmann.

Keller-Schneider, M., \& Hericks, U. (2021). Berufseinstieg von Lehrerinnen und Lehrern. In T. Hascher, U. Hericks \& W. Helsper (Hrsg.), Handbuch Schulforschung. Wiesbaden: Springer VS. https://doi. org/10.1007/978-3-658-24734-8.

Kiel, E. (2012). Sollen universitäre und zweite Ausbildungsphase stärker vernetzt oder stärker separiert werden? Mitteilungen des Deutschen Germanistenverbandes, 59(2), 182-184.

Köffler, N. (2015). Entwicklungsaufgaben im Lehramtsstudium. Bad Heilbrunn: Klinkhardt.

Košinár, J. (2014). Professionalisierungsverläufe in der Lehrerausbildung. Anforderungsbearbeitung und Kompetenzentwicklung im Referendariat. Opladen: Budrich. 
Košinár, J. (2018). Das Mentorat zwischen Individualisierung und Standardisierung - eine empirie- und theoriebasierte Konzeption. In C. Reintjes, G. Bellenberg \& G. im Brahm (Hrsg.), Mentoring und Coaching als Beitrag zur Professionalisierung angehender Lehrpersonen (S. 67-84). Münster: Waxmann.

Košinár, J. (2019a). Professionalisierung angehender Lehrpersonen - Herausforderungen und neue Erkenntnisse für die Schulpraktischen Studien. Zeitschrift der Bundearbeitsgemeinschaft für Schulpraktische Studien (BaSS), 13, 17-43.

Košinár, J. (2019b). Berufliche Identitätsbildung als Prozess und Entwicklungsaufgabe im Studium. In N. Safi, C. Bauer \& M. Kocher (Hrsg.), Lehrberuf: Vorbereitung, Berufseinstieg, Perspektiven. Beiträge aus der Professionsforschung (S. 83-98). Bern: hep.

Košinár, J. (2021). Typen der Anforderungsbearbeitung und Professionalisierungsverläufe im Referendariat. In J. Peitz \& M. Harring (Hrsg.), Das Referendariat (S. 93-104). Münster: Waxmann.

Košinár, J. (2021). Stabilität und Wandel ausbildungs- und berufsbezogener Orientierungen von Schweizer Primarschulstudierenden - Befunde einer dokumentarischen Längsschnittstudie. Beiträge zur Lehrerinnen- und Lehrerbildung. Eingereicht.

Košinár, J., \& Laros, A. (2018). Zwischen Einlassung und Vermeidung. Studentische Orientierungen im Umgang mit lehrberuflichen Anforderungen im Spiegel von Professionalität. In T. Leonhard, J. Košinár \& C. Reintjes (Hrsg.), Praktiken und Orientierungen in der Lehrerinnen- und Lehrerbildung (S. 157-174). Bad Heilbrunn: Klinkhardt.

Kuckartz, U. (2010). Einführung in die computergestützte Analyse qualitativer Daten. Wiesbaden: VS.

Leineweber, S. (2021). Studierende im Praktikum qualifiziert begleiten. Pädagogik, 02/2021, 38-42.

Mayring, P. (2010). Qualitative Inhaltsanalyse. Grundlagen und Techniken (11. erw. u. berarb. Aufl.). Weinheim und Basel: Beltz.

Ostermann, E. (2015). LehrerInwerden im Spannungsfeld subjektiver Erwartungen und objektiver Ausbildungsanforderungen. Professionsspezifische Entwicklungsaufgaben für Lehramtsstudierende. Bad Heilbrunn: Klinkhardt.

Pille, T. (2013). Das Referendariat Eine ethnographische Studie zu den Praktiken der Lehrerbildung. Bielefeld: transcript.

Schubarth, W., \& Wachs, S. (2020). Praxis im Vorbereitungsdienst. In C. Cramer, J. König, M. Rothland \& S. Blömeke (Hrsg.), Handbuch Lehrerinnen- und Lehrerbildung (S. 636-643). Bad Heilbrunn: Klinkhardt (utb).

Steinke, I. (2009). Gütekriterien qualitativer Forschung. In U. Flick, E. von Kardoff \& I. Steinke (Hrsg.), Qualitative Forschung. Ein Handbuch (S. 319-331). Reinbek: Rowohlt.

Terhart, E. (2011). Lehrerberuf und Professionalität: Gewandeltes Begriffsverständnis - neue Herausforderungen. In W. Helsper \& R. Tippelt (Hrsg.), Pädagogische Professionalität. Zeitschrift für Pädagogik, 57. Beiheft. (S. 202-224). Weinheim, Basel: Beltz.

Wittek, D. (2013). Heterogenität als Handlungsproblem. Entwicklungsaufgaben und Deutungsmuster von Lehrenden an Gemeinschaftsschulen. Opladen: Budrich. 